\title{
Game Transformation from Non-Augmented Reality to Augmented Reality
}

\author{
Chin-Tong Tan, Gi-Hyun Hwang, and Dae- Ki Kang ${ }^{*}$, Member, KIMICS
}

\begin{abstract}
The evolution of technology has lead gaming playable in augmented reality and more games are developed only for augmented reality. There are mainly no supportive reasons for game designed in nonaugmented reality and transforms to augmented reality. Genre of game played in augmented reality is always one of the defined game genres. In this paper, transformation process and effects of mobile games to augmented reality is discussed. Augmented reality game is recommended for mobile devices due to its portability. Modification for mobile games transformation including way of game rendering, game controlling, camera view controlling and game environment design are discussed. User experience is affected after the game transformed to augmented reality because of the differences in game controlling. The presence of game in real world is increased due to the usage of actual view in game playing.
\end{abstract}

Index Terms - Augmented Reality, Game, Mobile Device, User Experience.

\section{INTRODUCTION}

MASSIVE amount of video games are created and each of them is grouped into different genre such as shooting, fighting, role-playing and etc. The genre of the game will remain the same when the platform is changed. For example, a game ported form a personal computer to a mobile device, although the platform is different, the gameplay and genre will still be the same as before.

With the improvement of technology, playing video games using a gaming controller with few buttons is not the only way for gaming anymore. Loads of enhancement is done to the typical gaming controller to increase the interaction between the player and the game such as a motion detect controller. Subsequently, computer vision is also used with camera as another form of controller for gaming purpose. For smartphone and other mobile devices such as tablet computer, touchscreen is part of the possible interactions in gaming. Since the topic discussed is about augmented reality, camera is the main hardware for the discussion.

The main motivation of this discussion is about one

\footnotetext{
Manuscript received April 26, 2011; revised May 8, 2011; accepted May 28, 2011.

Chin-Tong Tan is with the Faculty of Information Technology, Multimedia University, Cyberjaya, Malaysia. (Email: johnny.tan.shinto88@gmail.com)

* Corresponding author (Email: dkkang@dongseo.ac.kr)
}

gameplay design that is playable in two different environments. The two environments indicated are game that is in non-augmented reality and augmented reality. Every single game developed before the introduction of augmented reality can be modified to augmented reality game.

Alternative incentive for the discussion is to support the recycle of video games. Mobile phone is evolved from merely a phone that can only do telephone calls to a gaming device. For games that used to be played with keypad or keyboard can be redeveloped again on new smartphones in augmented reality with camera or accelerometer installed. Games used to be on the top of game chart can be replayed again in augmented reality with different controlling system.

The user experience of the game will not be equal albeit the same gameplay is used. The way of controlling the game is changed, so as the way player play the game. In augmented reality, part of the virtual environment will be substitute to the real environment. Besides, the key to control the camera view in the game will be replaced to what the actual camera hardware detects. Amendment of actual surrounding to game can bring players to the next level of the gaming domain.

In the entire discussion, smartphone with camera mounted will be the main gaming device as it is portable and usually installed with powerful processor. Transformation within the same gaming console platform will be discussed due to porting issue of different programming languages and license issue for most of the games. This discussion included the similarities and differences between non-augmented reality game and augmented reality, by what method can the transformation be done to preserve the gameplay, and in what manner does the transformation affect the user experience.

\section{METHODOLOGY}

Since the discussion does not apply to any platform and method, specification of the technologies used and the justification for the usage is defined in this section. The actual implementation of the technologies is discussed in Section III.

To identify whether is this transformation noteworthy, experiments can be done to users from different skill of gaming, from newbie to expect. Method like examination of players reasoning can be done [3]. User experience can 
be extracted directly from the player in this method. But the obtaining of user experience is not included in the discussion.

\section{A. Virtual Reality and Augmented Reality}

In case of maximize the experience of gaming, why don't a game be developed in fully in virtual reality instead? One of the reasons is that the system for virtual reality is too expensive to be used by conventional player. Even at the standard of current technology, it is very hard to have a human to fully adapt to the virtual reality. A very powerful processor to generate a fully three-dimensional environment is obligatory. A system that can react to player's response immediately is required for virtual reality as well [1]. Human gestures are very complex and arduous to be processed by a computer. It is also necessity for player to feel that they are completely exists in the world of virtual reality. Human uses their senses to understand the environment, therefore a virtual reality system that can excite these senses are essential. The main problem to this is that nobody wants to be injured. Obviously, current technology could not fulfill the requirements to build a virtual reality game yet.

For the hindrances in virtual reality system, virtual reality is not convincing and substituted with augmented reality. Augmented reality for gaming would blend both the real world and virtual game world together. At the time when game is inherent into the real world, player will feel the presence of games in actual life. There are few requirements needed in order to use augmented reality. One of these is a monitor to show the projection of virtual objects. Additional requirement for augmented reality is a sensor to detect the environment. Without the sensor, there is no way the system could recognize the exact location to render the virtual object. The sensor can either be a camera or an accelerometer.

As an alternative to detect the environment with the sensor, a tracker (or marker) will be used to assist the detection of the environment. The tracker is an image contains feature points to let the detector recognize it. The augmented reality system will decode the tracker and tell the object renderer on where should the rendering occurred. Detecting a tracker is resource consuming because feature points on the tracker are geometry invariance or even photometric invariance [4]. A processor with better processing power is suggested so that the detection of tracker will run smoother. Since a tracker is used for environment detection, a camera will be used.

\section{B. Mobile Device}

A smartphone is an acceptable device for augmented reality as it has a screen to work as the monitor to display the enhanced scenery and a camera to detect the tracker. Smartphone built-in with a powerful processor is desirable for tracker detection. Other mobile devices with these features are also acceptable. Mobile device is chose to replace computer as its portability. A mobile device is freely movable with the camera and the monitor while this is hard to apply to a computer where the camera is possible to move easily but not the display. Camera view from the mobile will be the similar to a digital camera, whatever displayed on the screen reflected the view where the back of the screen faced. The view captured by the camera will be replaced with virtual object rendered and displayed on the screen of mobile device. Compare to a personal computer, drawbacks found for mobile devices are limited processing power and limited number of buttons for game controlling. Only restricted games can be designed and workable in mobile device with these drawbacks.

\section{GAME TRANSFORMATION}

Changes to be done on game transformation from nonaugmented reality to augmented reality are discussed in this section. Part A will deliberate what kind of game is transformable and to what form they will become. Next will be the controller changes from old platform to new platform included the changes of mobile devices design. Alteration of camera view in augmented reality is discussed in Part C. Differences of gaming environment before and after the transformation is mentioned subsequently followed by enhancement in user experience in the last section.

\section{A. Type of Game}

Any genre can be transformed into augmented reality platform subjected to how can it be done. Since the game is going to be played in augmented reality, the game objects are expected to be in three-dimensional. However the presence of the game in real world would not be less although the game is rendered in two-dimensional. Generally, the game genre does not affect the transformation of the game much.

The implementation of games into augmented reality will be different for two and three-dimensional game. For three-dimensional game, the object rendering is direct rendered on top of the real environment similar to implementation of other games or applications in augmented reality. Approach to apply two-dimensional game to augmented reality is even simpler. The whole window of the game is moved to the real environment in the form of a frame. The window in the real world works as part of the display of the game. Figure 1 below illustrated how does the two-dimensional game look like in augmented reality. Tic-tac-toe is the two-dimensional game that overwrites the tracker behind.

Since the changes for two-dimensional game is not noteworthy after transform into augmented reality, somehow it can be used as a single window to be shared 
among few players or even observers. More people sharing the same screen will be merrier than a small mobile screen that is visible by people surrounding it. This is similar to the existence of virtual sharing television in the real world.

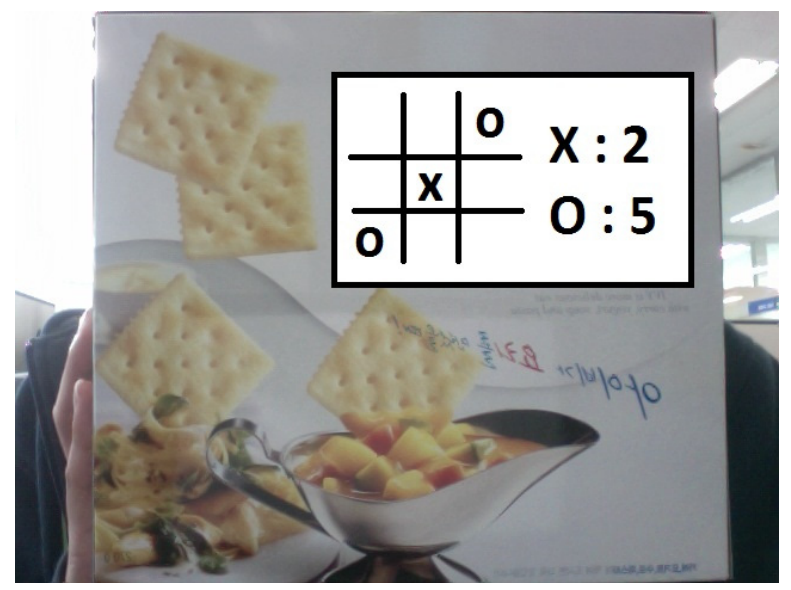

Fig. 1. Two-dimensional game rendered in augmented reality.

\section{B. Controller}

A lot of smartphones are designed without physical keypad or keyboard anymore. These keys are moved to the touchpad where they will only appear on the screen when they are needed. The main problem for the appearances of keypad or keyboard is that part of the screen will be filled. Significant view of the games might eventually be blocked. The usage of the keypad or keyboard in game controlling should be replaced to controlling game via gesture on the touchscreen. This is applied while a game is reapplied from an older device. New method to control the game should be design since the keyboard is no longer used. The game controlling system should be the same either they are played fully in augmented reality or not but the viewing system will somehow affect the design of controller and will be discussed in the next subtopic.

\section{Camera View}

Viewing system for both non-augmented reality game and augmented reality game will be different and controlling system for viewing will not be the same. For the game that is played without augmented reality, the camera view of the game will either be controlled by game itself via algorithm or controlled by the players. The controlling system will make no difference if the camera view of the non-augmented reality game is controlled by the game. Extra action to control the camera is not needed. On the other hand, extra commands are needed for player to alter the camera view. When the game started in non-augmented reality, the camera view is always set at the point where the game should begin.
For games played in augmented reality, no extra commands are needed as the movement of the physical camera will altered the view accordingly. It is a direct control on the physical object instead of tapping or dragging the touchscreen on commanding where to move. The game will somehow be more complicated as player needs to memorize more commands on gaming and camera controlling to play the game. However, the camera view is not directed to the point where the game should start. Hint should always be given so that the player would not be lost.

Point of view of some game genre does affect the transformation too. As known, first person shooting game uses the perspective of first person and some simulation games like The Sims uses the point of view from third person. For both of the game genres listed as examples, players themselves are the one who taking fully control over the camera view. In the case of first person shooter, camera controlling is vital as it is the movement of camera equal to the movement of character in the game. In augmented reality, there should be instructions defined to the player on how should they alter the camera along the tracker to have the character moved. Meanwhile, moving the camera along the tracker is how the player moves their view along the world for games in third person perspective. This approach is straightforward and everybody knows this from instinct.

\section{Environment}

How would the game environment be formed in augmented reality? It will be form either on top of the tracker or direct overlapping it. At the moment the orientation of the tracker is identified through the features point, these details will be used for rendering object. Boundary for the game environment will be the location where the tracker cannot be seen. This is not necessary for the non-augmented reality game for the reason that the world of the games is always in sight under no circumstances.

The game environment is also affected by the dimension whether is it in two-dimensional or threedimensional. Nothing is changed for two-dimensional game whether they are in augmented reality or not. Unlike two-dimensional game, objects in three-dimensional game will always be displayed in actual figure when the camera is moved. Whenever the camera is moved closer to the tracker, objects will be seen bigger as it became nearer to the camera. In augmented reality, background of the game that are not crucial will be ignore and replaced with real environment surrounding the tracker. Figure 2 below exemplified that no artificial background such as ground is needed. Ground of the game is replaced by the tracker existed in real world. In contrast, virtual background is designed to replace the environment of game that is played without augmented reality. 


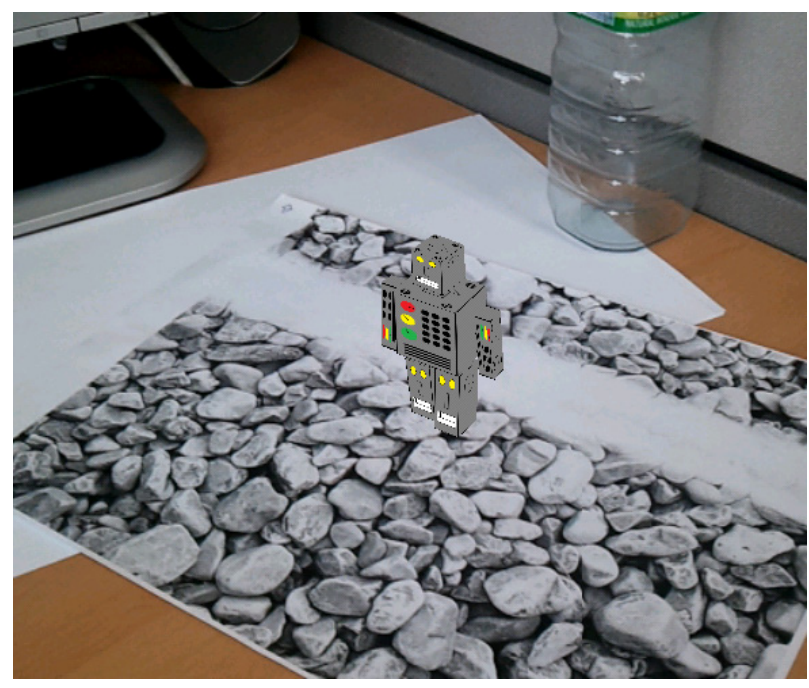

Fig. 2. Augmented reality example of virtual robot stands on actual ground.

\section{E. User Experience}

Given that the gameplay is not modified in the transformation to augmented reality, it is always presume to be meaningless and the transformation would not made much contribution. For game developers, this process may be considered as time and manpower consuming. Actually, all of the transformation is meant for better user experience while playing video games. If porting a game from old platform without changing anything, the game might be perceived as the same. Game repackaging to augmented reality can bring the epic moments of players towards that game again. It can even cause gaming in augmented reality a trend as gaming in augmented reality is not famous yet.

Possible actions on the touchscreen as game instruction will be less for augmented reality game. The reason is that the instructions for camera controlling are substituted to direct camera moving. Less command to be used in the game will ease the players in command memorization. For people who are not used to video games, they can concentrate more on the action commands. Controlling the camera will be depends on instinct instead. Newbie will get used of the game without controlling direct control of the view at learning stage. While newbie become better, they can play the game in non-augmented reality and learn to control the camera view later. For gaming expect, they can choose either to play the game in augmented reality or not.

Playing games in augmented reality will have the sense of the game presence in real world. Some games are better to be played like they are really existed in the real world. For example, a pet breeding game, everybody hope to breed a real pet than a virtual one. Augmented reality will enhance the feel of breeding virtual pet since they can be seen in real environment with the support of augmented reality. There are times when the games cannot be played in augmented reality such as missing of tracker or tracker cannot be detected. Switching can be done for games that are designed for both augmented reality and nonaugmented reality. More choices are given to player to increase the excitement of the video games.

\section{CONCLUSIONS}

Other than user experience, there are more reasons for games to be developed in both non-augmented reality and augmented reality, such as increasing the target market to users that could not support the game in augmented reality. Else, a gameplay that must be played in both non-augmented reality and augmented reality environment can be designed. Augmented reality will be improved to another standard once it is fully utilized, so as the standard of gaming.

\section{ACKNOWLEDGMENT}

This research was conducted in TPR3311 industrial training program of Multimedia University, Malaysia and a research program of Dongseo University's Ubiquitous Appliance Regional Innovation Center supported by the grants from Ministry of Knowledge Economy of the Korean government. (No. B0008352)

\section{REFERENCES}

[1] J. Vallino, "Interactive Augmented Reality," Ph.D. dissertation, Department of Computer Science, University of Rochester, Rochester, NY, April 1998.

[2] Qualcomm Incorporate. (2011). Augmented Reality: QCAR Developer Guide [Online]. Available: http://developer.qualcomm.com/dev/augmented-reality.

[3] S. Song, J. Lee, and I. Hwang, "A New Framework of Usability Evaluation for Massively Multi-player Online Game: Case Study of "World of Warcraft" Game" Proc. the 12th International Conference on Human-Computer Interaction, vol. 4, pp. 341-350, July 2007.

[4] R. Szeliski, Computer Vision: Algorithms and Applications, 1st ed. Springer, 2010.

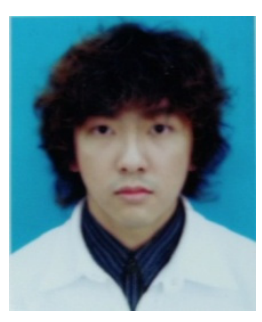

Chin-Tong Tan is born in Malaysia at the year of 1988. He is currently undergoing B.S. degree in computer science from Multimedia University, Malaysia. His interests include 3D computer graphics, logic programming and human computer interaction.

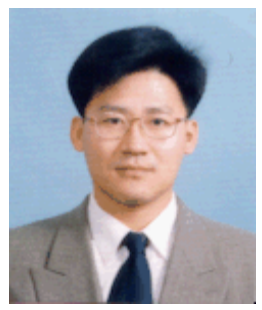

Gi-Hyun Hwang received his M.S. and Ph.D. in Electrical Engineering, Pusan National University. He is currently a professor at Dongseo University. His research interested lies in applications of intelligent control to power system, RFID, and Embedded System. E-mail: hwanggh@gdsu.dongseo.ac.kr 


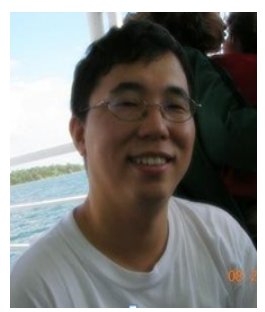

Dae-Ki Kang is an assistant professor at Dongseo University in South Korea. He was a senior member of engineering staff at the attached Institute of Electronics \& Telecommunications Research Institute in South Korea. He earned a $\mathrm{PhD}$ in computer science from Iowa State University in 2006 . His research interests include social network services, mac hine learning, relational learning, statistical graphical models, metaheuristics, ontology learning, Tower of Hanoi, multimedia systems, intrusion detection, Web firewall, and computer vision. Prior to joining Iowa State, he worked at a Bay-area startup company and at Electronics and Telecommunication Research Institute in South Korea. He received a science master degree in computer science at Sogang University in 1994 and a bachelor of engineering (BE) degree in computer science and engineering at Hanyang University in 1992. 